CP, 2018, Vol.7 - No15, pp. 247/263 ISSN 2014-6752. Girona (Catalunya). Universitat de Girona. VAZQUEZ-SANDE, Pablo; PÉREZ-GARCÍA, Antonia; GARCÍA-ABAD, Lito: La función de relaciones públicas vista por estudiantes universitarios gallegos

Recibido: 14/05/2018 - Aceptado: 15/10/2018

\title{
La función de relaciones públicas vista por estudiantes universitarios gallegos
}

\section{Public relations role perceived by Galician university students}

\author{
Autores: \\ Pablo Vazquez-Sande \\ Profesor departamento Ciencias de la Comunicación \\ Universidad de Santiago de Compostela. \\ http://orcid.org/0000-0001-5081-385X \\ Antonia Pérez-García \\ https://orcid.org/0000-0002-4655-8830 \\ Profesora titular de Grado en Turismo \\ Escuela Universitaria de Turismo CENP \\ Lito García-Abad \\ https://orcid.org/0000-0002-4974-0224 \\ Profesor Publicidad y Relaciones Públicas CESUGA
}

\section{Resumen}

La evolución de la percepción del concepto de Relaciones Públicas desde su tradicional uso vulgar, aún vigente en la actualidad (Magallón, 1998-1999 y 2006), hasta su consolidación como disciplina autónoma y ciencia social (Noguero, 1988; Castillo, 2009; Rojas, 2012; L'Etang, 2009) debería caminar junto a la profesionalización del concepto y del perfil profesional (Armendáriz, 2015) entre los estudiantes universitarios que incluyen en su programa formativo disciplinas encuadrables en esa área de conocimiento. El estudio realizado entre varios colectivos de universitarios gallegos arroja, sin embargo, unos resultados que siembran dudas sobre si realmente estamos avanzando en la aceptación social de una ciencia y profesión que debe incardinarse en la realidad de las empresas actuales y futuras.

Palabras clave: Relaciones Públicas; percepción; comunicación; universidad.

\section{Abstract}

Concept evolution of Public relations from its traditional vulgar use, currently in use (Magallón, 1998-1999 and 2006), until its consolidation as an autonomous discipline and social science (Noguero, 1988; Castillo, 2009; Rojas, 2012; L'Etang, 2009) should go in hand with the professionalization of the concept and professional profile (Armendáriz, 2015) among undergraduate students that include in their formal program disciplines that fit into that area of knowledge. The research conducted among several groups of Galician undergraduate students show, nevertheless, doubts about if we are really improving in the social acceptance of a science and profession that must be incardinated in the reality of current and future companies.

Keywords: Public Relations; perception; communication; university. 


\section{Introducción}

Mucho se ha discutido entre profesionales y académicos sobre el concepto y naturaleza de las relaciones públicas, cuál es su objeto científico y cuál es la realidad social de sus profesionales. Tampoco podemos obviar que, en el afán e intento de explicar el concepto de relaciones públicas y en su construcción disciplinar, hayan surgido ciertas tensiones paradigmáticas, tal y como afirman Sadi y Méndez (2015), sumado a que, aunque se ha escrito mucho sobre relaciones públicas, el acervo de conocimientos teóricos es reducido y se ha visto afectado por paradigmas dominantes.

Otra cuestión importante a destacar en la dialéctica sobre contenidos y significados del propio concepto de relaciones públicas, son los errores que se pueden hallar en los libros de texto de esta disciplina, libros de texto traducidos de obras originales, mayormente procedentes de EEUU. Traducciones sin adaptación a contextos locales que limitan su significado y aplicación llevando a graves errores de interpretación (Mckie, D. y Munshi, D., 2011).

Por otro lado, y no menos importante, está el hecho de que el concepto siga teniendo connotaciones negativas. Aced, C. (2013) hace hincapié en ello apoyándose en un estudio de Dircom en 2012 en el que se afirma que 6 de cada 10 dircom españoles opina que tiene claras connotaciones negativas, lo que representa en España un 14\% más que en el resto de Europa.

En este estudio se ha preguntado a los futuros profesionales de diversas áreas de conocimiento (ADE, Empresariales, Turismo, Traducción, Publicidad y Relaciones Públicas, Humanidades y Ciencias de la Comunicación), en las que al menos disponen de una asignatura vinculada con la Comunicación Organizacional, qué entienden ellos por Relaciones Públicas. Puntualizando que parte de esos alumnos ya se habían enfrentado al menos a esa asignatura de Comunicación Organizacional en la que se incluyen nociones básicas sobre la disciplina objeto de análisis, y otros aún no lo habían hecho, con lo que se podía observar, a su vez, si había alguna diferencia entre las percepciones de los alumnos en un caso y en el otro.

La selección de los centros se ha realizado por criterios meramente prácticos: en ellos desempeñan los autores su labor docente por lo que era más fácil realizar las encuestas diseñadas al efecto. Los cuatro centros utilizados son gallegos y dos de ellos son públicos, mientras que los otros dos son centros adscritos, uno a una universidad pública y otro a una universidad privada.

\subsection{Marco teórico}

El objeto de esta investigación parte de la previa definición del concepto de relaciones públicas. Estamos ante una disciplina consolidada, donde prácticamente ya no existen diferencias ontológicas apreciables. De ahí que la investigación en relaciones públicas no se centre en su concepto sino en sus distintas aplicaciones, por lo que la literatura actual lo único que hace es recoger los conceptos asentados desde el último cuarto del siglo XX. Así, Wilhelm, Ramírez y Sánchez (2009) recoge la literatura de los últimos treinta años; Míguez (2011) incluye como referencia más reciente en su bibliografía un artículo de 2006, y García Nieto (2011) se refiere a un trabajo de ese mismo año siendo el siguiente más reciente entonces de 2007. Y todos ellos, por supuesto, recogiendo la literatura del último cuarto de siglo. 
CP, 2018, Vol.7 - No15, pp. 247/263 ISSN 2014-6752. Girona (Catalunya). Universitat de Girona. VAZQUEZ-SANDE, Pablo; PÉREZ-GARCÍA, Antonia; GARCÍA-ABAD, Lito: La función de relaciones públicas vista por estudiantes universitarios gallegos

Recibido: 14/05/2018 - Aceptado: 15/10/2018

\title{
La función de relaciones públicas vista por estudiantes universitarios gallegos
}

\section{Public relations role perceived by Galician university students}

\author{
Autores: \\ Pablo Vazquez-Sande \\ Profesor departamento Ciencias de la Comunicación \\ Universidad de Santiago de Compostela. \\ http://orcid.org/0000-0001-5081-385X \\ Antonia Pérez-García \\ https://orcid.org/0000-0002-4655-8830 \\ Profesora titular de Grado en Turismo \\ Escuela Universitaria de Turismo CENP \\ Lito García-Abad \\ https://orcid.org/0000-0002-4974-0224 \\ Profesor Publicidad y Relaciones Públicas CESUGA
}

\section{Resumen}

La evolución de la percepción del concepto de Relaciones Públicas desde su tradicional uso vulgar, aún vigente en la actualidad (Magallón, 1998-1999 y 2006), hasta su consolidación como disciplina autónoma y ciencia social (Noguero, 1988; Castillo, 2009; Rojas, 2012; L'Etang, 2009) debería caminar junto a la profesionalización del concepto y del perfil profesional (Armendáriz, 2015) entre los estudiantes universitarios que incluyen en su programa formativo disciplinas encuadrables en esa área de conocimiento. El estudio realizado entre varios colectivos de universitarios gallegos arroja, sin embargo, unos resultados que siembran dudas sobre si realmente estamos avanzando en la aceptación social de una ciencia y profesión que debe incardinarse en la realidad de las empresas actuales y futuras.

Palabras clave: Relaciones Públicas; percepción; comunicación; universidad.

\section{Abstract}

Concept evolution of Public relations from its traditional vulgar use, currently in use (Magallón, 1998-1999 and 2006), until its consolidation as an autonomous discipline and social science (Noguero, 1988; Castillo, 2009; Rojas, 2012; L'Etang, 2009) should go in hand with the professionalization of the concept and professional profile (Armendáriz, 2015) among undergraduate students that include in their formal program disciplines that fit into that area of knowledge. The research conducted among several groups of Galician undergraduate students show, nevertheless, doubts about if we are really improving in the social acceptance of a science and profession that must be incardinated in the reality of current and future companies.

Keywords: Public Relations; perception; communication; university. 


\section{Introducción}

Mucho se ha discutido entre profesionales y académicos sobre el concepto y naturaleza de las relaciones públicas, cuál es su objeto científico y cuál es la realidad social de sus profesionales. Tampoco podemos obviar que, en el afán e intento de explicar el concepto de relaciones públicas y en su construcción disciplinar, hayan surgido ciertas tensiones paradigmáticas, tal y como afirman Sadi y Méndez (2015), sumado a que, aunque se ha escrito mucho sobre relaciones públicas, el acervo de conocimientos teóricos es reducido y se ha visto afectado por paradigmas dominantes.

Otra cuestión importante a destacar en la dialéctica sobre contenidos y significados del propio concepto de relaciones públicas, son los errores que se pueden hallar en los libros de texto de esta disciplina, libros de texto traducidos de obras originales, mayormente procedentes de EEUU. Traducciones sin adaptación a contextos locales que limitan su significado y aplicación llevando a graves errores de interpretación (Mckie, D. y Munshi, D., 2011).

Por otro lado, y no menos importante, está el hecho de que el concepto siga teniendo connotaciones negativas. Aced, C. (2013) hace hincapié en ello apoyándose en un estudio de Dircom en 2012 en el que se afirma que 6 de cada 10 dircom españoles opina que tiene claras connotaciones negativas, lo que representa en España un 14\% más que en el resto de Europa.

En este estudio se ha preguntado a los futuros profesionales de diversas áreas de conocimiento (ADE, Empresariales, Turismo, Traducción, Publicidad y Relaciones Públicas, Humanidades y Ciencias de la Comunicación), en las que al menos disponen de una asignatura vinculada con la Comunicación Organizacional, qué entienden ellos por Relaciones Públicas. Puntualizando que parte de esos alumnos ya se habían enfrentado al menos a esa asignatura de Comunicación Organizacional en la que se incluyen nociones básicas sobre la disciplina objeto de análisis, y otros aún no lo habían hecho, con lo que se podía observar, a su vez, si había alguna diferencia entre las percepciones de los alumnos en un caso y en el otro.

La selección de los centros se ha realizado por criterios meramente prácticos: en ellos desempeñan los autores su labor docente por lo que era más fácil realizar las encuestas diseñadas al efecto. Los cuatro centros utilizados son gallegos y dos de ellos son públicos, mientras que los otros dos son centros adscritos, uno a una universidad pública y otro a una universidad privada.

\subsection{Marco teórico}

El objeto de esta investigación parte de la previa definición del concepto de relaciones públicas. Estamos ante una disciplina consolidada, donde prácticamente ya no existen diferencias ontológicas apreciables. De ahí que la investigación en relaciones públicas no se centre en su concepto sino en sus distintas aplicaciones, por lo que la literatura actual lo único que hace es recoger los conceptos asentados desde el último cuarto del siglo XX. Así, Wilhelm, Ramírez y Sánchez (2009) recoge la literatura de los últimos treinta años; Míguez (2011) incluye como referencia más reciente en su bibliografía un artículo de 2006, y García Nieto (2011) se refiere a un trabajo de ese mismo año siendo el siguiente más reciente entonces de 2007. Y todos ellos, por supuesto, recogiendo la literatura del último cuarto de siglo. 
Las relaciones públicas son el objeto de una disciplina que se apoya en las ciencias sociales tal y como afirmó en su momento Bernays (1990), considerado el padre de las relaciones públicas como ciencia social moderna. Se destaca que utilizan medios propios de investigación científica y de análisis, modelos y técnicas que le permiten alcanzar el objetivo del diálogo y comprensión entre los diferentes públicos, tanto en los espacios privados como en la esfera pública.

Al abordar la expresión "relaciones públicas" se hace imprescindible indagar en los dos términos que la componen: "relaciones" y "públicas". Noguero (1995) argumenta que la expresión se podría analizar desde dos niveles: el primer nivel haría referencia al factum del mundo de los fenómenos como un ser humano, una institución o una empresa; en este primer nivel de análisis el autor lo relaciona con el hecho técnico del sujeto promotor de las relaciones públicas. El segundo nivel haría referencia al actum con relación a la realidad operativa de la actividad o práctica de las relaciones públicas.

Por otro lado, Noguero (1995: 52-55), también plantea abordar otro tipo de análisis de la expresión relaciones públicas que marcarían tres estadios diferentes: la "acepción anecdótica", que estaría relacionado con la procedencia del término anglosajón Public Relations de fácil manejo en el lenguaje y de uso recurrente, lo que le ha llevado a establecer variados usos y fines que lo aleja de la función profesional verdadera y alberga un importante confusionismo; la "acepción culta de la expresión", en la que existen similitudes y diferencias encontradas en cuanto al propio significado de la expresión que podemos encontrar en diferentes documentos divulgativos, tipo diccionarios de diversa índole, y la "acepción técnico-académica" del conocimiento específico de las relaciones públicas.

\subsubsection{Naturaleza y definición de Relaciones Públicas}

Son muchas las definiciones de relaciones públicas (Noguero (1990: 65-66) que se pueden hallar en la revisión de la literatura, unas con más rigor desde el punto de vista científico y/o profesional y otras con menos acierto y más controversia (diccionarios al uso, diccionarios especializados en las ciencias sociales, revistas especializadas, manuales, entre otros).

También Xifra (2003: xvii) alude a la existencia de esta ambigüedad en la mayoría de las definiciones de relaciones públicas, confusionismo que afecta a los teóricos y profesionales de las relaciones públicas, sumado al desconocimiento de objetivos y contenidos de esta disciplina por parte de la sociedad en general; problema que también se extiende al marco teórico, reflejo de una "fragilidad intelectual de profesionales y universitarios a la hora de generar unas bases sólidas que pongan freno a usurpadores terminológicos".

Una de las primeras definiciones aparece en el Public Relations News (citado en Grunig y Hunt, 2000: 55) en su primer boletín de noticias semanal sobre esta actividad, definición que ha resistido el paso del tiempo y a la que más tarde Marston (1983: 6), en un intento de hacerla más precisa y correcta, añadió el concepto "comunicación": "Las relaciones públicas son la función gerencial que evalúa las aptitudes públicas, identifica las políticas y procedimientos de una organización con el interés público, y ejecuta un programa de acción y comunicación para ganar aceptación y entendimiento públicos".

Arceo Vacas J.L. (1988:21) habla nuevamente de filosofía gerencial, desde la óptica de la em- 
Las relaciones públicas son el objeto de una disciplina que se apoya en las ciencias sociales tal y como afirmó en su momento Bernays (1990), considerado el padre de las relaciones públicas como ciencia social moderna. Se destaca que utilizan medios propios de investigación científica y de análisis, modelos y técnicas que le permiten alcanzar el objetivo del diálogo y comprensión entre los diferentes públicos, tanto en los espacios privados como en la esfera pública.

Al abordar la expresión "relaciones públicas" se hace imprescindible indagar en los dos términos que la componen: "relaciones" y "públicas". Noguero (1995) argumenta que la expresión se podría analizar desde dos niveles: el primer nivel haría referencia al factum del mundo de los fenómenos como un ser humano, una institución o una empresa; en este primer nivel de análisis el autor lo relaciona con el hecho técnico del sujeto promotor de las relaciones públicas. El segundo nivel haría referencia al actum con relación a la realidad operativa de la actividad o práctica de las relaciones públicas.

Por otro lado, Noguero (1995: 52-55), también plantea abordar otro tipo de análisis de la expresión relaciones públicas que marcarían tres estadios diferentes: la "acepción anecdótica", que estaría relacionado con la procedencia del término anglosajón Public Relations de fácil manejo en el lenguaje y de uso recurrente, lo que le ha llevado a establecer variados usos y fines que lo aleja de la función profesional verdadera y alberga un importante confusionismo; la "acepción culta de la expresión", en la que existen similitudes y diferencias encontradas en cuanto al propio significado de la expresión que podemos encontrar en diferentes documentos divulgativos, tipo diccionarios de diversa índole, y la "acepción técnico-académica" del conocimiento específico de las relaciones públicas.

\subsubsection{Naturaleza y definición de Relaciones Públicas}

Son muchas las definiciones de relaciones públicas (Noguero (1990: 65-66) que se pueden hallar en la revisión de la literatura, unas con más rigor desde el punto de vista científico y/o profesional y otras con menos acierto y más controversia (diccionarios al uso, diccionarios especializados en las ciencias sociales, revistas especializadas, manuales, entre otros).

También Xifra (2003: xvii) alude a la existencia de esta ambigüedad en la mayoría de las definiciones de relaciones públicas, confusionismo que afecta a los teóricos y profesionales de las relaciones públicas, sumado al desconocimiento de objetivos y contenidos de esta disciplina por parte de la sociedad en general; problema que también se extiende al marco teórico, reflejo de una "fragilidad intelectual de profesionales y universitarios a la hora de generar unas bases sólidas que pongan freno a usurpadores terminológicos".

Una de las primeras definiciones aparece en el Public Relations News (citado en Grunig y Hunt, 2000: 55) en su primer boletín de noticias semanal sobre esta actividad, definición que ha resistido el paso del tiempo y a la que más tarde Marston (1983: 6), en un intento de hacerla más precisa y correcta, añadió el concepto "comunicación": "Las relaciones públicas son la función gerencial que evalúa las aptitudes públicas, identifica las políticas y procedimientos de una organización con el interés público, y ejecuta un programa de acción y comunicación para ganar aceptación y entendimiento públicos".

Arceo Vacas J.L. (1988:21) habla nuevamente de filosofía gerencial, desde la óptica de la em- 
presa o institución, y traduce el término filosofía en acciones de comunicación de tipo persuasivo de todas las clases: interpersonal, colectiva o masiva, predominantemente informativa.

Una de las definiciones más extensas y completas es la de Rex Harlow (1976:36) donde combinó 472 definiciones, después de revisar documentos y hablar con profesionales de las relaciones públicas, y donde recoge todas las variables que entrarían en juego en el concepto, desde concebirlas como una función de dirección anticipadora de tendencias hasta la aplicación de la ética en el mundo empresarial (citado en Grunig y Hunt, 2000: 53).

Aunque al principio se alude a la falta de unitarismo conceptual, Simon (1994:20) y Cutlip y Center (2001:36), entre otros autores, coinciden en que todas estas definiciones tienen componentes en común y resumen los elementos coincidentes en muchas de ellas, como se aprecia en el siguiente cuadro:

Tabla 1. Resumen comparativo de diferentes definiciones entre Simon y Scott Cutlip y Allen Center, basándose en diferentes autores

\begin{tabular}{|c|c|}
\hline Simon $\mathrm{R}$. & Cutlip y Center \\
\hline $\begin{array}{l}\text { Un esfuerzo planificado o función } \\
\text { administrativa. }\end{array}$ & $\begin{array}{l}\text { Actúan de acuerdo a un programa } \\
\text { planificado y sostenido como parte de } \\
\text { la dirección de una organización. }\end{array}$ \\
\hline $\begin{array}{l}\text { La relación entre organización y } \\
\text { sus públicos. }\end{array}$ & $\begin{array}{l}\text { Tratan de las relaciones entre una } \\
\text { organización y sus públicos. }\end{array}$ \\
\hline $\begin{array}{l}\text { Evaluación de las actitudes y } \\
\text { opiniones del público. }\end{array}$ & $\begin{array}{l}\text { Supervisan el conocimiento, opiniones, } \\
\text { actitudes y comportamiento dentro y } \\
\text { fuera de la organización. }\end{array}$ \\
\hline $\begin{array}{l}\text { Evaluar las políticas, } \\
\text { procedimientos y acciones de una } \\
\text { organización en cuanto que se } \\
\text { relacionan con esos públicos de la } \\
\text { organización. }\end{array}$ & $\begin{array}{l}\text { Analizar el impacto de estrategias, } \\
\text { procedimientos y acciones sobre los } \\
\text { públicos. }\end{array}$ \\
\hline $\begin{array}{l}\text { Pasos adoptados para asegurar } \\
\text { que esas políticas, procedimientos } \\
\text { y acciones sean de interés público } \\
\text { y socialmente responsables. }\end{array}$ & $\begin{array}{l}\text { Ajustan aquellas estrategias, } \\
\text { procedimientos y acciones que puedan } \\
\text { estar en conflicto con el interés } \\
\text { público o la supervivencia de la } \\
\text { organización. }\end{array}$ \\
\hline $\begin{array}{l}\text { Ejecución de un programa de } \\
\text { acción, de comunicación o ambos. }\end{array}$ & $\begin{array}{l}\text { Establecen y mantienen comunicación } \\
\text { bidireccional entre la organización y } \\
\text { sus públicos. }\end{array}$ \\
\hline \multirow[t]{3}{*}{$\begin{array}{l}\text { Desarrollo de simpatía, buena } \\
\text { voluntad, entendimiento y } \\
\text { aceptación como el principal } \\
\text { objetivo que se busca con la } \\
\text { actividad de las relaciones } \\
\text { públicas. }\end{array}$} & $\begin{array}{l}\text { Aconsejan a la dirección sobre el } \\
\text { establecimiento de nuevas estrategias, } \\
\text { procedimientos y acciones que puedan } \\
\text { ser mutuamente beneficiosas para la } \\
\text { organización y sus públicos (están } \\
\text { hablando de buscar el entendimiento, } \\
\text { comprensión y la aceptación). }\end{array}$ \\
\hline & $\begin{array}{l}\text { Realizan cambios específicos en el } \\
\text { conocimiento, opiniones, actitudes y } \\
\text { comportamiento dentro y fuera de la } \\
\text { organización. }\end{array}$ \\
\hline & $\begin{array}{l}\text { Impulsan nuevas relaciones } 0 \\
\text { mantienen las existentes entre una } \\
\text { organización y sus públicos. }\end{array}$ \\
\hline
\end{tabular}

Fuente: Elaboración propia. 


\subsubsection{Función de las Relaciones Públicas}

Para comprender el contenido y las funciones que pueden abarcar las relaciones públicas es necesario precisar el papel que ejercen estas, abordando sus objetivos y las áreas de actuación. Puede que existan ideas confusas al respecto, especialmente en lo que a la práctica de la profesión se refiere, y que siga siendo un tema de discusión a nivel académico, pero hay una serie de objetivos y funciones en los que, una mayoría de los teóricos y profesionales de las relaciones públicas, coinciden en estar de acuerdo.

Marston (1983: 154-162) sugirió que pueden ser definidas a partir de cuatro funciones específicas: Investigación, Acción, Comunicación y Evaluación, conocido como el modelo R-A-C-E (Research, Action, Communication, Evaluation) que en realidad es en forma de espiral cerrada porque cada función lleva a la siguiente por lo que la evaluación -última etapa de la espiraltambién sirve como investigación para programas futuros.

Black (1991:37) afirma que las relaciones públicas tienen una doble función: ejecutiva y consultora. Winner (1991) argumenta que las relaciones públicas desempeñan una función que puede variar considerablemente de una organización a otra, aunque puede existir una función básica que permanece igual, la ejecución de la función varía mucho de una empresa privada, por ejemplo, a una organización pública, de una empresa local a una nacional, debido a factores intrínsecos propios de cada una. En cada situación específica, los públicos estarán compuestos por aquellos grupos con los que la organización lidia.

Figura 1. Modelo de la función de las Relaciones Públicas en una organización.

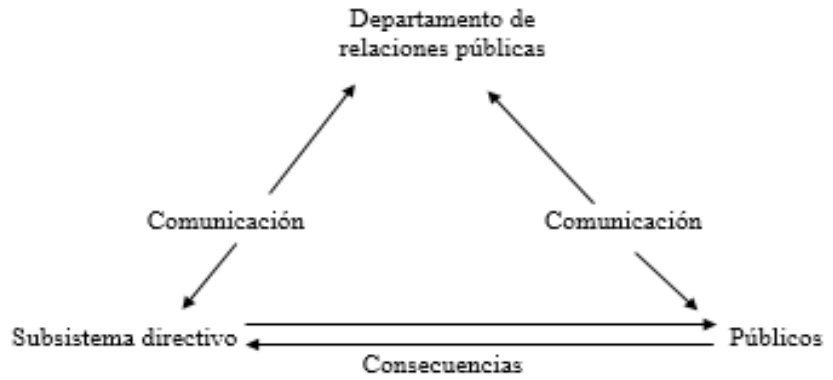

Fuente: Grunig, J. y Hunt, T. (2000:58)

Grunig y Hunt (2000) parten del hecho de que las relaciones públicas funcionan como un subsistema directivo o de gestión, ayudando a la dirección a planificar y evaluar las actividades de comunicación global en cualquier organización. Cada subsistema afectaría a los demás y, a su vez, a la organización total porque son subsistemas que interactúan. Ellos proponen un prototipo de la función de las relaciones públicas en una organización que sería el que se expone a continuación (p. 59): 
Tabla 2. Análisis crítico de Porto Simôes sobre los pre-paradigmas que explican las funciones de Relaciones Públicas

Enfoque estructural

Son una función o subfunción administrativa.

Son un sistema institucional.

Son un subsistema de apoyo.

Enfoque administrativo

Son la administración del conflicto.

Son la administración de la controversia.

Son la administración de los problemas emergentes (Issue Management).

Enfoque filosófico-práctico

Son una filosofía social.

Son la proyección de las relaciones humanas.

Son $90 \% R$ (realización) y $10 \%$ P (publicidad).

Enfoque comunicacional

Buscan establecer y mantener la comprensión mutua.

Son un medio de comunicación.

Buscan integrar intereses (públicosy/o privados).

Son una vía de doble tránsito o circulación (bilateralidad de informaciones).

Enfoque político

Son una política de puertas abiertas (buscando generar confianza).

Son una casa de vidrio (transparencia en la comunicación).

Enfoque psicológico

Buscan formar imagen.

Buscan formar concepto (sobre la organización).

Buscan formar actitud (como proximidad a la praxis de la política).

Buscan formar opinión pública.

Buscan obtener buena voluntad.

Enfoque mercadológico

Son un instrumento de marketing.

Enfoque personalizado

Son contacto 
Por otro lado, Porto Simôes (1993) hace una crítica sobre las diferentes funciones de las relaciones públicas, a partir de las proposiciones teóricas o pre-paradigmas de otros autores que podemos resumir en el siguiente cuadro. A ese respecto aclara que, aisladamente, estos enfoques son insuficientes para explicar la actividad de las relaciones públicas, ya que tienen una visión parcial de las mismas:

Pero ¿dónde encaja el departamento de relaciones públicas y sus funciones en el organigrama organizacional? Una evidencia clara es que el rol de las relaciones públicas, dentro de la organización, depende en gran medida de la percepción que tenga la dirección sobre éstas (Wilcox, et al. 2012). Según estos autores, cada vez es más habitual que el profesional de relaciones públicas dependa directamente de dirección, ya que las relaciones públicas comienzan en la alta dirección, y abogan por que la filosofía de relaciones públicas sea una práctica de la política de toda la organización.

\subsubsection{Disciplinas y actividades afines a las Relaciones Públicas 1.1.3.1. Relaciones Públicas y Publicidad}

Rojas Orduña (2012) destaca que la primera controversia entre ambas disciplinas es el propio concepto, en inglés "publicits" se refiere a los profesionales de las relaciones públicas y en algunos manuales se ha realizado una traducción errónea al castellano.

González Martín (1996) declara que la publicidad y las relaciones públicas pueden estar dentro de un mismo programa de comunicación persuasiva porque pueden complementar la acción comercial, las relaciones públicas se gestionan para la empresa/institución y la publicidad para los productos y servicios. Las relaciones públicas estarían relacionadas con la comunicación institucional para dar a conocer, promover y defender a una empresa y, aunque la publicidad también puede cumplir estos objetivos, las relaciones públicas se basan en una comunicación multidireccional y, por el contrario, la publicidad lo hace "al margen de avatares competitivos del bien, servicio o institución" (p. 17).

Arceo Vacas J.L. (1988:51) define la publicidad como "una comunicación persuasiva colectiva, fundamentalmente de masas, de carácter comercial e impulsada por un anunciante cuyo fin es la difusión de sus bienes y servicios para una posterior venta o contratación de éstos". Dirá que un tipo de publicidad, llamada publicidad institucional o corporativa y que apareció después de la crisis del petróleo de 1973, tiene mucho que ver con las relaciones públicas.

Wilcox, Cameron y Xifra (2012) parten de un planteamiento integrador de las tres disciplinas: relaciones públicas, publicidad y marketing, desde el cual la organización puede alcanzar mejor sus objetivos, teniendo claro el cometido de cada una de ellas.

Con una visión más radical, en marcar la diferencia entre ambas disciplinas, están Ries A. y Ries L. (2003: 25), ellos exponen su regla general: "Nunca hacer publicidad hasta que se haya explotado las posibilidades de las RR.PP., y seguirán manifestando su planteamiento al respecto diciendo: "La publicidad no significa creación de marcas. Éste es el papel y la función de las RR.PP. La publicidad significa mantenimiento de marcas" (p. 219), es decir, que la función de la publicidad es reforzar las ideas y conceptos creados o gestionados por las relaciones públicas, ya que son éstas las que contribuyen a implantar las credenciales de la empresa. 


\subsubsection{Relaciones Públicas y Propaganda}

Herreros Arconada (1989) hace un análisis exhaustivo entre publicidad y propaganda porque encuentra que es ahí donde radican las mayores confusiones, por lo menos en el terreno práctico, no tanto teórico, llamando publicidad a lo que en rigor es propaganda o viceversa.

\section{Tabla 3. Diferencias entre Publicidad y Propaganda}

\begin{tabular}{|l|l|}
\hline \multicolumn{1}{|c|}{ PUBLICIDAD } & \multicolumn{1}{|c|}{ PROPAGANDA } \\
\hline Forma de comunicación persuasiva. & Forma de comunicación persuasiva. \\
\hline $\begin{array}{l}\text { Persigue influir en la conducta } \\
\text { comercial del receptor. }\end{array}$ & $\begin{array}{l}\text { Persigue influir en la conducta política } \\
\text { del receptor. }\end{array}$ \\
\hline $\begin{array}{l}\text { Difunde sus mensajes utilizando los } \\
\text { medios de comunicación masiva y y } \\
\text { otros propios. }\end{array}$ & $\begin{array}{l}\text { Difunde sus mensajes utilizando los } \\
\text { medios de comunicación masiva, los } \\
\text { publicitarios y otros que le son propios. }\end{array}$ \\
\hline $\begin{array}{l}\text { Paga el tiempo o el espacio de los } \\
\text { medios que emplea. }\end{array}$ & $\begin{array}{l}\text { No siempre paga el tiempo y el espacio } \\
\text { de los medios que emplea. }\end{array}$ \\
\hline $\begin{array}{l}\text { El emisor se identifica con cada } \\
\text { mensaje. }\end{array}$ & $\begin{array}{l}\text { El emisor no siempre se identifica con } \\
\text { cada mensaje. }\end{array}$ \\
\hline $\begin{array}{l}\text { La estructura del mensaje es } \\
\text { reconocida como publicitaria por el } \\
\text { receptor. }\end{array}$ & $\begin{array}{l}\text { La estructura de los mensajes puede } \\
\text { tomar cualquier forma } \\
\text { comunicación. }\end{array}$ \\
\hline $\begin{array}{l}\text { Las consecuencias de la acción son } \\
\text { generalmente irrelevantes para el } \\
\text { receptor ya que se circunscribe a su } \\
\text { área económica. }\end{array}$ & $\begin{array}{l}\text { Las consecuencias afectan a las } \\
\text { actitudes fundamentales del individuo } \\
\text { y repercuten en la estructura político- } \\
\text { social. }\end{array}$ \\
\hline $\begin{array}{l}\text { En todas sus manifestaciones adopta } \\
\text { formas específicas. }\end{array}$ & $\begin{array}{l}\text { Puede adoptar todo tipo de formas de } \\
\text { comunicación. }\end{array}$ \\
\hline
\end{tabular}

Fuente: Herreros Arconada (1989:95)

Una frase de Xifra (2005:53) resume claramente la diferencia entre relaciones públicas, publicidad y propaganda: "mientras la publicidad y la propaganda se dirigen a las emociones, las relaciones públicas se dirigen a la razón".

\subsubsection{Relaciones Públicas y Publicity}

Creemos imprescindible matizar el concepto de publicity, por su cercanía a las relaciones públicas. Cutlip y Center (2001:42) definen la publicity como "la información proporcionada por una fuente externa que los medios de comunicación utilizan por tener valor informativo. Se trata de un método no controlado de difundir mensajes, ya que la fuente no paga a los medios por dicha difusión"; explican que en los primeros tiempos las relaciones públicas fue- 
ron básicamente publicity y que, actualmente, existe un número importante de empresas que limitan la actividad de las relaciones públicas a la publicity, es decir, al envío de información a los medios de comunicación, no entendiendo que la actividad de las relaciones públicas es mucho más amplia que la publicity. A esta reducción de las relaciones públicas, aluden Wilcox, Cameron y Xifra (2012), se añade la confusión errónea de equiparar la publicity con la publicidad, aclarando que la publicidad paga el espacio y el tiempo mientras que la publicity, esencialmente, es la cobertura informativa.

\subsubsection{Relaciones Públicas y Protocolo}

En ocasiones es lógico que estas dos disciplinas, relaciones públicas y protocolo, colaboren porque ambas comunican, establecen un diálogo y crean imagen porque, resumiendo, buscan el reconocimiento hacia la organización o institución que representan. Salla y Ortega (2008: 169) argumentan que "el protocolo tiene como objetivo principal la proyección de la imagen pública de la organización y es una función natural del profesional de relaciones públicas". Y siguiendo en esta misma línea, Otero Alvarado (2004) expone que los acontecimientos especiales de relaciones públicas se mueven en el marco del ceremonial y el protocolo, son eventos íntimamente relacionados con la "reputación corporativa" de la empresa, en el sentido de que buscan el reconocimiento de los públicos hacia el comportamiento de la organización; por lo tanto han de trabajar conjuntamente en busca de una mayor eficacia (Palencia-Lefler, 2011)

El concepto de protocolo tiene una variada y amplia significación pero en síntesis se refiere a la organización de actos a los que suele afectar a determinadas personas que, por su rango social o cargo, serán acomodadas en determinado lugar que les corresponde.

\subsubsection{Relaciones Públicas y Marketing}

Pero sin duda, el debate más encarnizado se enraíza frente al marketing. Muchos autores tratan este punto; algunos otorgan al marketing una entidad superior, lo que ha llevado a generar cierta confusión al respecto. Para Rosenberg (citado en Arceo Vacas, 1988: 49) es una actividad comercial que acelera el movimiento de bienes y servicios desde el fabricante al consumidor a través de las políticas mix -publicidad, promoción, distribución, transporte, política de producto y de venta, investigación, etc. Ries A. y Ries L. (2003) argumentan al respecto que muchas empresas ponen el énfasis en la publicidad, equiparando publicidad a marketing, partiendo de la idea de que las relaciones públicas son una disciplina secundaria; ello, dicen estos autores, es un error grave porque la publicidad carece de credibilidad, cuestión que no ocurre por igual con las relaciones públicas. Por su lado, Cutlip y Center (2001) aluden a este confusionismo creyendo que, en parte, es debido a que el marketing utiliza acepciones erróneas como "marketing social", "comunicación de marketing" o incluso "relaciones públicas de marketing", llevando a la conclusión falsa de que no hay diferencia entre ambas. Y Arceo Vacas, A. (1995: 126) dirá sobre Kotler que éste desconoce el objetivo real de las relaciones públicas al "supeditarlas al marketing" porque las incluye en el contexto de los servicios y productos y que, por ello, no es la persona más indicada para "teorizar sobre relaciones públicas". Sobre ello, declara Fraser (2002), el marketing crea y conserva el mercado para los productos y servicios, en cambio las relaciones públicas crean y mantienen un entorno sociable en la organización y el éxito del marketing depende de las políticas sociales planificadas y gestionadas de las relaciones públicas. 


\section{Metodología}

El estudio que se presenta se basa en una investigación exploratoria con metodológica cuantitativa a través de la aplicación de una encuesta estructurada que responde al objetivo principal de partida: conocer la percepción del concepto de relaciones públicas entre los universitarios gallegos.

El estudio se centra en una muestra de estudiantes universitarios correspondientes a la Universidad de A Coruña, Universidad de Santiago y CESUGA (provincias de A Coruña y Lugo). Se toma como población objeto de estudio a futuros profesionales de diversas áreas de conocimiento ( $A D E$, Empresariales, Turismo, Traducción, Publicidad y Relaciones Públicas, Humanidades y Ciencias de la Comunicación), en las que al menos disponen de una asignatura vinculada con la Comunicación Organizacional.

Se parte de la siguiente premisa: parte de esos alumnos ya se habían enfrentado al menos a esa asignatura de Comunicación Organizacional en la que se incluyen nociones básicas sobre la disciplina objeto de análisis, y otros aún no lo habían hecho bien porque acaban de incorporarse a la universidad, bien porque en sus planes de estudio todavía no estaban matriculados en la asignatura en cuestión.

El período de aplicación de las encuestas fue enero de 2018. A todos ellos se les facilitó un cuestionario estructurado dividido en dos bloques: variables sociodemográficas para determinar el perfil del estudiante, y variables relacionadas con la medición de nociones sobre el concepto de relaciones públicas y percepción de otras disciplinas afines. En total se recogieron 182 encuestas entre todos los centros de estudios seleccionados por motivos de operatividad.

Se trata de una primera exploración con ánimo de continuidad en el tiempo en la que se busca reflejar los resultados de base que sirvan para medir la evolución temporal de esa percepción sobre los mismos centros y tipología de alumnos. Por tanto, no se buscaba en este momento obtener resultados certeros y representativos del total de la población universitaria gallega, sino la base 100 sobre la que se irá midiendo en el futuro la evolución de las opiniones de un colectivo plural de estudiantes universitarios.

En cuanto al cuestionario, sobre un primer borrador se aplicó un proceso de corrección de posibles sesgos antes de la entrega del definitivo. En su versión última se establecen un total de 13 ítems, algunos de ellos para marcar una sola opción y otros con posibilidad de opción múltiple.

Se incluyen dos preguntas abiertas: ¿Qué es un profesional de las relaciones públicas? ¿Cuáles son sus funciones? y ¿Cuáles son las funciones de un profesional de la publicidad? Estas preguntas abiertas, que se categorizan y tabulan independientemente, se plantean con el objetivo de conocer con mayor exactitud las diferencias que creen existir entre estos dos tipos de profesionales de la comunicación.

\section{Resultados}

El perfil de los encuestados revela que el $87,77 \%$ de los alumnos ya han cursado previamente alguna asignatura relacionada con la comunicación. La mayoría son mujeres (67,03\%), siguiendo la tendencia habitual en el panorama universitario español, y la edad media global de quienes participan en la investigación es 19,87 años. 
En cuanto al área en la que enmarcarían la comunicación organizacional (gráfico 1), menos de la mitad aciertan a situarla entre las relaciones públicas. Así, de las 155 respuestas válidas obtenidas para esta pregunta, solo 70 (un 45,16\%) vinculan ambas disciplinas. Resulta significativo que en titulaciones menos especializadas en las relaciones públicas el grado de asociación es mayor: por ejemplo, entre los estudiantes de Ciencias de la Cultura el $83,33 \%$ de las respuestas establecen esa adscripción, mientras que entre los de Periodismo solo el 26,19\% de los alumnos encajan la comunicación organizacional en las relaciones públicas.

\section{Gráfico 1. Disciplina que enmarcaría dentro del área de comunicación organizacional}

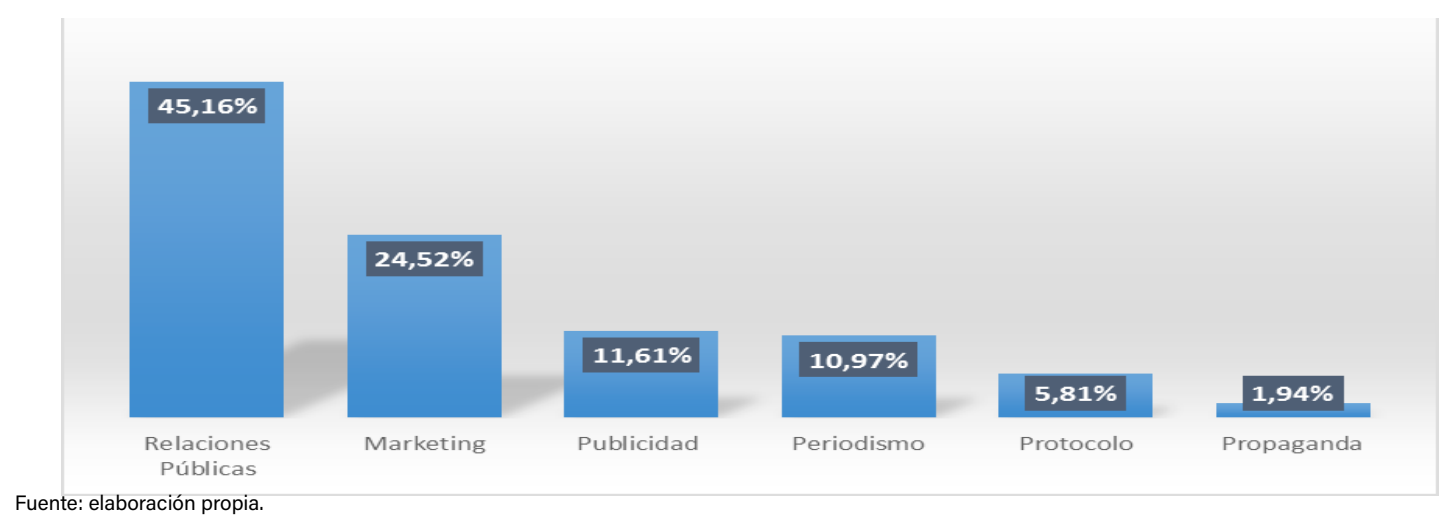

Volviendo al conjunto de la muestra, el 24,52\% considera que la comunicación organizacional debe encuadrarse en el ámbito del marketing, mientras que el $11,61 \%$ la sitúa en la publicidad, el 10,97\% en el periodismo, el 5,81\% en el protocolo y, finalmente, el 1,94\% en la propaganda. El cuestionario pedía a los participantes una definición del profesional de las relaciones públicas y de sus funciones. Las respuestas obtenidas fueron diversas (se categorizaron 24 ítems), aunque la más frecuente vinculaba al trabajador de este ámbito con la comunicación desde un punto de vista global (27 respuestas). En segundo lugar, 23 alumnos asociaron al profesional de las relaciones públicas con la gestión de la imagen, frente a los 19 que subrayaron su papel de enlace o de actor con la sociedad. Aunque minoritarias, alguna respuesta asocia al Relaciones Públicas a la investigación (un estudiante), a la venta del producto (cinco alumnos) o a la captación de clientes (dos).

Consecuentemente, las funciones que se le asignan con mayor frecuencia a este profesional son la gestión de relaciones con el entorno (15 respuestas), seguida de la gestión de la imagen (13) y de la comunicación (12). Entre las respuestas espontáneas brindadas por los encuestados, se alude, entre otras muchas cuestiones, también a la gestión de la promoción (9), de la venta de productos (8) y de la información (4).

Mientras, al profesional de la publicidad lo vinculan, principalmente, a la gestión de las campañas de publicidad (36 respuestas), de la publicidad, actividades, elementos o técnicas publicitarias (32) y a la gestión de la promoción (25). Resulta singular las relaciones que entablan algunos estudiantes entre la publicidad y las relaciones públicas, ya que, por ejemplo, uno de los encuestados señala que "la publicidad es un recurso o instrumento que ayuda al Relacio- 
nes Públicas", mientras que otro argumenta que "no las considero muy diferentes, [se trata de] coordinar la comunicación de su entidad", lo que parece desdibujar las fronteras entre ambas disciplinas.

\section{Gráfico 2. Objetivos más relevantes de las relaciones públicas}

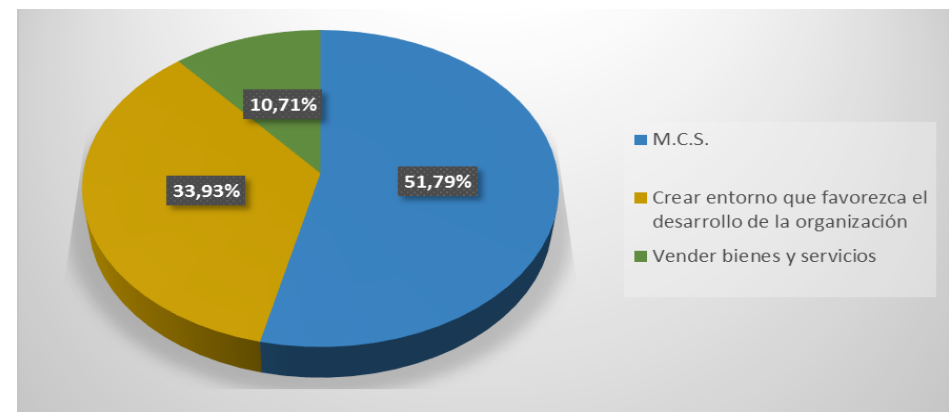

Fuente: elaboración propia

Al margen de ello, otra de las cuestiones nucleares de la encuesta pivotaba en torno a los objetivos que persiguen las relaciones públicas (gráfico 2). Respecto a ella, la mayoría de alumnos perpetúan la consideración de que el trabajo de un profesional de esta área se ciñe fundamentalmente a los medios de comunicación, respuesta por la que se decantaron el $51,79 \%$ de los encuestados.

Por el contrario, el 33,93\% opinan que la finalidad de su trabajo ha de ser la de crear un entorno que favorezca el desarrollo de la organización en cuestión. Por titulaciones, los de Ciencias de la Cultura son quienes más aciertan al marcar esta opción el $50 \%$ de los encuestados. También los de Publicidad y Relaciones Públicas la señalan en un $44,44 \%$ de sus respuestas, así como el $38 \%$ de los de Periodismo.

Son minoría (un 10,71\%) los que creen que la principal finalidad de un Relaciones Públicas es la de vender bienes y servicios. De hecho, ningún encuestado de las titulaciones de Publicidad y RRPP y de Ciencias de la Cultura considera que esta sea la tarea de un profesional de este ámbito.

Gráfico 3. Nivel al que tiene que estar la gestión de RR.PP.

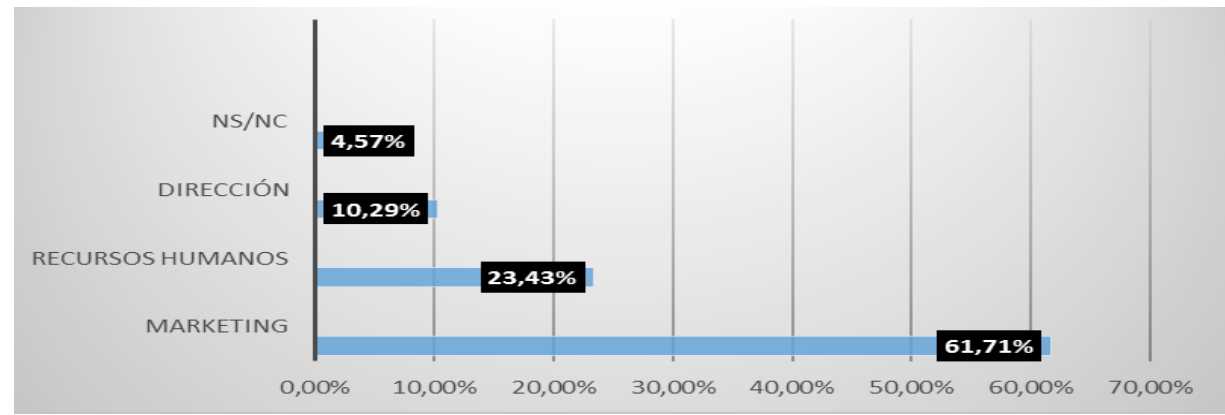

Fuente: elaboración propia 
Frente a la superación de esa función instrumentalista comercial de las relaciones públicas, lo que todavía no se ha corregido es el nivel al que ha de situarse este departamento en las organizaciones (gráfico 3). Así, el 61,71\% cree que deberían adscribirse al marketing frente al $23,43 \%$ que lo ubican en recursos humanos. De este modo, la opción correcta desde el punto de vista de la literatura científica (a nivel directivo) solo alcanza el 10,29\% de las respuestas, mientras que el $4,57 \%$ de los encuestados aseguran desconocerlo.

La tendencia es similar entre los estudiantes de todas las titulaciones al decantarse mayoritariamente por el área de marketing. Solo los estudiantes de Publicidad y Relaciones Públicas quiebran esa tónica ( $50 \%$ sitúan las relaciones públicas a nivel directivo, frente al $42,31 \%$ que las encuadran en el marketing y el 7,69\% en los recursos humanos).

Gráfico 4. Cualidades del profesional de las RR.PP.

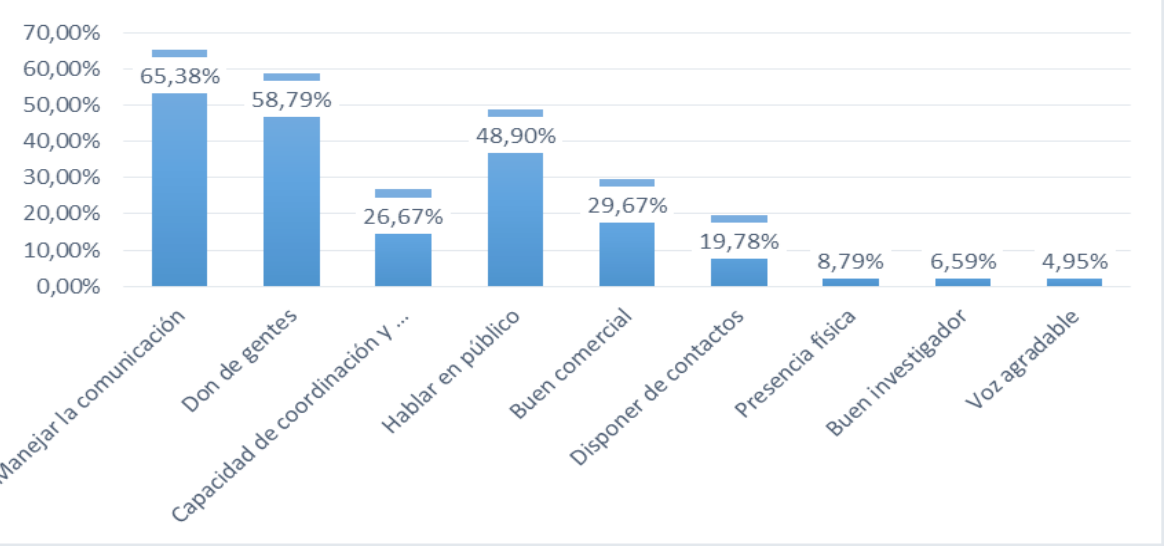

Fuente: elaboración propia

Respecto a las cualidades que debe poseer un profesional de las Relaciones Públicas (gráfico 4), las tres que más se repiten son la de manejar la comunicación en todas sus áreas (65,38\% de los encuestados), tener don de gentes (58,79\%) y capacidad de coordinación y organización (53,85\%). A estas les siguen la cualidad de saber hablar en público $(48,9 \%)$, de ser buen comercial $(29,67 \%)$ y de disponer de muchos contactos $(19,78 \%)$. Por el contrario, por debajo de la barrera del $10 \%$ se quedan la buena presencia física $(8,79 \%)$, la capacidad de ser buen investigador $(6,59 \%)$ y, finalmente, poseer una voz agradable $(4,95 \%)$.

En relación al mercado laboral de un trabajador de Relaciones Públicas (pregunta de respuesta múltiple), el $82,42 \%$ identifica la planificación y gestión de la comunicación, seguida del 54,4\% que apunta a la relación con los medios. Quienes todavía los vinculan al ámbito comercial representan un nada despreciable $34,62 \%$, al igual que los que todavía asocian estos profesionales a ser representantes de discotecas o entidades de espectáculos $(23,63 \%)$, en menor medida $(6,04 \%)$ azafatas.

Por titulaciones, la mayor unanimidad a la hora de señalar la planificación y gestión de comunicación se encuentra entre los estudiantes de Ciencias de la Cultura (100\%), los de Publicidad y Relaciones Públicas (93,33\%) y los de Periodismo (87,04\%). 
Los resultados obtenidos entre los alumnos de Turismo y de Empresariales que aún no habían dado la asignatura de Técnicas de Comunicación (primer curso) arrojaron un porcentaje del $19 \%$ de alumnos que se refirieron a alguna de esas características fundamentales recogidas por Cutlip y Center (2001), mientras que el restante $81 \%$ lo hizo a aspectos muy parciales vinculados a la comunicación. Sin embargo, el porcentaje no mejora sustancialmente entre los alumnos que ya han impartido asignaturas de comunicación y cuyos grados no pertenecen al ámbito de la comunicación.

\section{Gráfico 5. Características de las RR.PP.}

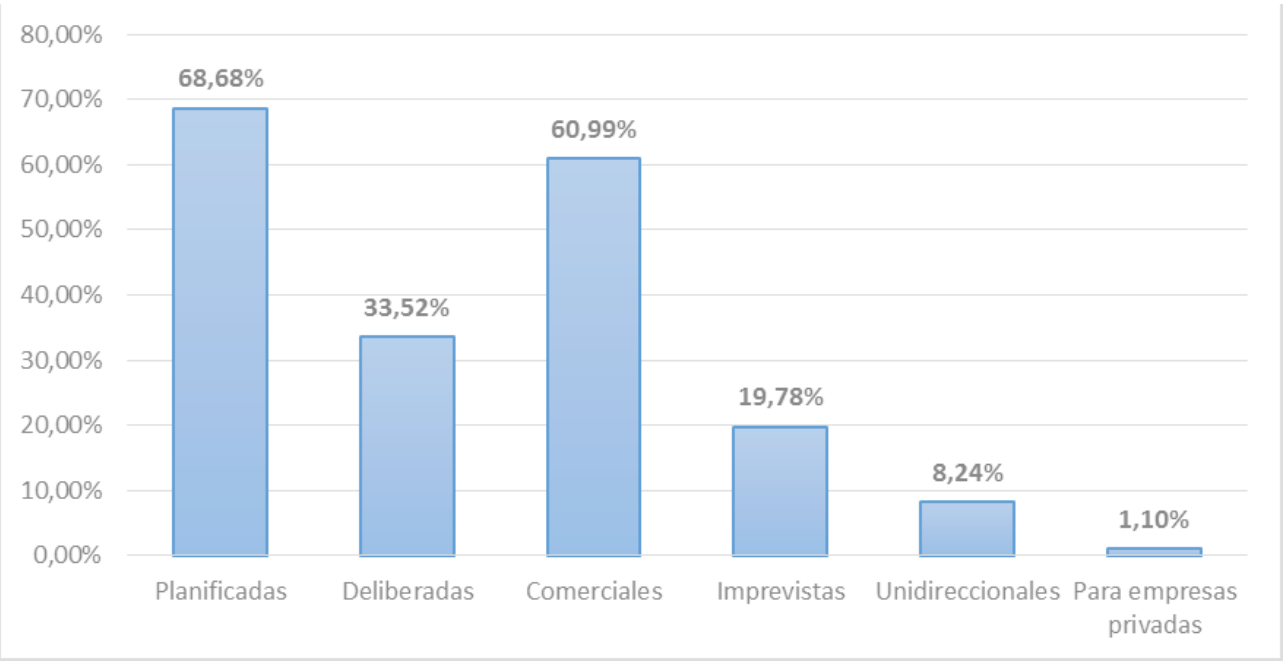

Fuente: elaboración propia

Por último, en cuanto a las características de las relaciones públicas (gráfico 5), un 68,68\% acierta al señalar que deben ser planificadas, mientras que solo uno de cada tres considera que tengan que ser deliberadas (33,52\%). Resulta llamativo que el 60,99\% de los encuestados insistan en apreciar una veta comercial, al igual que el hecho de que el $19,78 \%$ interpreten que las Relaciones Públicas son imprevistas, el $8,24 \%$ opinen que son unidireccionales o que un $1,1 \%$ las vea propias solo para empresas privadas.

\section{Discusión y conclusiones}

La percepción de los estudiantes sobre las relaciones públicas también se centran en la función de la Publicity, como en el estudio de Moreno, Navarro y Humanes (2014) sobre la imagen de la función de relaciones públicas entre las empresas. Curiosamente, estas autoras inciden en que una de las acciones que podrían contribuir al fortalecimiento de la profesión sería la formación en esta área. Pero como se ha podido comprobar en este estudio, el hecho de haber recibido nociones básicas sobre la disciplina de relaciones públicas en el contenido de alguna asignatura de comunicación, no es motivo como para tener demasiado claro qué es un profesional de las relaciones públicas.

Con todo, se ha detectado una cierta superación de la consideración de que un profesional de Relaciones Públicas se ocupa directamente de la venta de productos y servicios. Pese a 
que todavía pervive entre los encuestados la idea de que este trabajador contribuye a esta finalidad, diversas respuestas (por ejemplo, la del objetivo principal de un Relaciones Públicas o su mercado laboral) parecen sugerir un cierto optimismo en consonancia con los postulados teóricos que separan las relaciones públicas de la dimensión estrictamente comercial.

En el polo opuesto, pese a que los alumnos asocian las relaciones públicas a la planificación, sorprende el poco respaldo que prestan a la necesidad de investigar (es el segundo rasgo menos importante con el que caracterizan a estos profesionales).

Se ha detectado también que los encuestados no sitúan al departamento de Relaciones Públicas en el nivel directivo, tal y como debería de ser (Wilcox et al, 2012, Clutip y Center, 2001 y Grunig J y Grunig L., 1991). Mayoritariamente lo asocian al departamento de Recursos Humanos; creemos que por su conexión con la idea de interacción entre personas que subyace bajo ambos conceptos, y al departamento de Marketing, por su clara relación con el enfoque comercial y mercantilista en que se ve envuelto el concepto "Relaciones Públicas".

Una línea futura de investigación sería trabajar con una muestra mayor de estudiantes, ampliar la representatividad de los centros de estudio universitarios y contrastar si la evolución de esa percepción universitaria corre pareja de la evolución de los profesionales ya ejercientes en sus respectivas áreas de competencia, así como comprobar si la evolución de la percepción de esa función corre pareja a la empresarial con la progresiva introducción de los egresados en las organizaciones.

\section{Referencias}

- ACED, C. (2013). Relaciones públicas 2.0. Cómo gestionar la comunicación corporativa en el entorno digital. Barcelona: Editorial UOC S.L., Colección Comunicación.

- ARCEO VACAS, A. (1995). Las relaciones públicas y el marketing: crítica a los planteamientos de Kloter. Questiones publicitarias: Revista internacional de Comunicación y Publicidad, no 4, 121-133. Recuperado el 20 de febrero de 2018 de https://tinyurl.com/ydbmp8ws

- $\quad$ ARCEO VACAS, J. L. (1988). Fundamentos para la teoría y técnica de las Relaciones Públicas. Barcelona: Editorial PPU.

- ARMENDÁRIZ, R. (2015). El nuevo perfil del profesional de la Comunicación y las Relaciones Públicas. Una visión desde la perspectiva de mercado. Revista Internacional de Relaciones Públicas, no 9 Vol. V, (pp. 153-178). Recuperado e 17 de enero de 2018 https:// tinyurl.com/y7mf5rvw

- $\quad$ BERNAYS, L. E. L. (1990). Los últimos años. Radiografía de las Relaciones Públicas 19561986. Barcelona: ESRP-PPU.

- $\quad$ BLACK, S. (1991). Las relaciones públicas, un factor clave de gestión. Barcelona: Editorial Hispano Europea.

- CAStillo esparcia, A. (2009). Relaciones Públicas. Teoría e Historia. Barcelona: Editorial UOC. Primera Edición.

- CUTLIP SCOTT, M. y CENTER, A. (2000). Relaciones públicas eficaces. Clásicos de las Relaciones Públicas. Barcelona: Editorial Gestión 2000.

- $\quad$ FRASER, P. SEITEL. (2002). Teoría y práctica de las relaciones públicas. Madrid: Editorial Pearson Educación. Octava edición.

- GARCÍA NIETO, M. T. (2011). La investigación en las relaciones públicas: tendencias y para- 
dojas. Actas Icono 14, no A6, pp. 139-158, ISBN. 1697-8293.

- $\quad$ GONZÁLEZ MARTíN, A. (1996). Teoría general de la publicidad. Madrid: Fondo de Cultura Económica de España. Primera edición.

- $\quad$ GRUNIG, J. E. y HUNT, T. (2000). Dirección de relaciones públicas. Barcelona: Editorial Gestión 2000. Primera edición.

- $\quad$ GRUNIG, J., GRUNIG, L. y APARECIDA-FERRARI, M. (2015). Perspectivas de las Relaciones Públicas: resultados del Excellence Study para la comunicación en las organizaciones. Revista Mediterránea de Comunicación, 6 (2), 9-28. Recuperado el 20 de febrero de 2018 en https://tinyurl.com/yc95s9cw

- HERREROS ARCONADA, M. (1989). Teoría y técnica de la propaganda electoral (formas publicitarias). Colección Comunicación y Relaciones Públicas. Barcelona: PPU.

- L'ETANG, J. (2009). Relaciones Públicas. Conceptos, práctica y crítica. Barcelona: Editorial UOC. Colección Comunicación. Primera edición.

- MAGALLÓN, S. (1998-1999). Las relaciones públicas en el ámbito de las ciencias sociales y su papel en las organizaciones. Revista Universitaria de Publicidad y Relaciones Públicas, n० 5-6, 139-146.

- (2006). Concepto y elementos de las relaciones públicas. Anàlisi, 34, 103-109

- MARSTON, J. (1983). Relaciones públicas modernas. México: Editorial McGraw-Hill.

- MCKIE D. y MUNSHI, D. (2011). Reconfigurar las relaciones públicas. Ecología, equidad y empresa. Colección Comunicación. Barcelona: Editorial UOC, S.L.

- MíGUEZ, GONZÁLEZ, M. I. (2011). La investigación sobre relaciones públicas en las revistas españolas de comunicación (2008-2010). Actas Icono 14, n A6, pp. 124-138, ISBN. 1697-8293.

- $\quad$ MORENO Ma A., NAVARRO, C. y HUMANES Ma L. (2014). El liderazgo en relaciones públicas y gestión de comunicación. Análisis cuantitativo de los factores de liderazgo en el sector España. Revista Palabra Clave, Vol. 17, n 3 (pp. 946-978), Universidad de la Sabana, Bogotá. Recuperado el 20 de febrero de 2018 en https://tinyurl.com/yb37mqoe

- NOGUero GRAU, A. (1988). Programación y técnicas de relaciones públicas. Colección Comunicación y Relaciones Públicas. Barcelona: Promociones y Publicaciones Universitarias.

- (1990). Relaciones Públicas e industria de la persuasión. Colección Comunicación y Relaciones Públicas. Barcelona: PPU.

- (1995). La función social de las relaciones públicas: historia, teoría y marco legal. Colección Comunicación y Relaciones Públicas. Barcelona: PPU.

- PALENCIA-LEFLER, M. (2011). 90 Técnicas de comunicación y relaciones públicas. Editorial Bresca (Profit Editorial S.L.)

- PORTO SIMÔES, R. (1993). Relaciones Públicas: función política en la empresa y en la institución pública. Barcelona: Consejo Superior de Relaciones Públicas de España. Tercera edición.

- $\quad$ RAYMOND, S. (1994). Relaciones públicas. Teoría y práctica. México: Limusa Noriega Ediciones.

- RIES, A. y RIES, L. (2003). La caída de la Publicidad y el auge de las Relaciones Públicas. Barcelona: Ediciones Urano S.A.

- ROJAS ORDUÑA, O.I. (2012). Relaciones Públicas: la eficacia de la influencia. Madrid: ESIC. Tercera edición.

- SADI. G. y MÉNDEZ. V. (2015). Una aproximación histórica al dominio intelectual de las 
Relaciones Públicas. Tensiones paradigmáticas en su construcción disciplinar. Revista Internacional de Relaciones Públicas, no 9, Vol. 5, 27-66. Recuperado el 20 de febrero de 2018 de https://tinyurl.com/ycc2yz7n

- $\quad$ SAlla GARCÍA, J. y ORTEGA SORIANO, J. (2008). Plan estratégico de Relaciones Públicas. Barcelona: Bosch Editor.

- WILCOX, D., CAMERON, G. y XIFRA, J. (2012). Relaciones Públicas. Estrategias y tácticas. Madrid: Pearson Educación, S.A. Décima edición ampliada.

- WILHELM, G., RAMÍREZ, F. y SÁNCHEZ, M. (2009). Las Relaciones Públicas: herramienta fundamental en la creación y mantenimiento de la identidad e imagen corporativa. Razón y Palabra, vol. 14, n 70 , noviembre-enero.

- WINNER, P. (1991). Gestâo moderna das relaçôes públicas. Manual de direcçâo eficaz. Estratégia da confiança. Colecçao Gestâo. Portugal: Ediçôes CETOP.

- XIFRA, J. (2003). Teoría y estructura de las Relaciones Públicas. Madrid: Editorial McGraw Hill. Primera edición.

- (2005). Planificación estratégica de las relaciones públicas. Barcelona: Editorial Paidós Ibérica S.A. Primera edición.

\section{CURRICULUM VITAE \\ Pablo Vázquez-Sande}

Doctor en Comunicación por la Universidad de Santiago de Compostela (USC), en la que defendió la tesis doctoral Storytelling personal en el relato político con la que obtuvo la máxima calificación de sobresaliente cum laude y varios premios internacionales.

Compatibiliza su trabajo académico como docente de varias asignaturas en la USC y en el Centro de Estudios Superiores Universitarios de Galicia (CESUGA) con la consultoría al frente de la agencia Atalaya Comunicación.

\section{Antonia Pérez García}

Profesora Titular de la Escuela Universitaria de Turismo CENP, adscrita a la Universidade da Coruña. Dra. por la Universidade da Coruña en Análisis Económico y Estrategia empresarial. Licenciada en Sociología por la Universidade da Coruña. Técnico Superior en Relaciones Públicas por la Escuela Superior de Relaciones Públicas, adscrita a la Universidad de Barcelona

\section{Lito García Abad}

Es profesor del grado de Publicidad y Relaciones Públicas en CESUGA (centro adscrito a la Universidad de San Jorge) y del grado de Turismo en la Escuela Universitaria de Turismo de A Coruña. Doctor en Ciencias de la Información por la Universidad Complutense de Madrid, Licenciado en Derecho por la UNED y Máster en Abogacía por la Universidad de Santiago de Compostela, actualmente es doctorando en Derecho y Ciencias Sociales por la UNED. 